\title{
Comparative Study of Pulmonary Function Tests in Women in Different Phases of Menstrual Cycle with and Without Contraceptive Pills
}

\author{
Dr. Raksha Hebbar.K \\ Department of Physiology, Vydehi Institute of Medical Sciences and Research Centre, Bangalore, India
}

\begin{abstract}
:
Background And Objectives: The relationship between the synthetic hormones and premenstrual asthma has long been debated with conflicting reports regarding the effects of estrogen and progesterone. Oral contraceptives appear to have beneficial effect on lung mechanics and the proposed mechanisms vary from ventilation stimulant effect of progesterone to the collagen increasing effect of estrogen. The objective of this study was to evaluate a cross section of combined oral contraceptive pill users with healthy controls in different phases of menstrual cycle with computerized spirometry and to compare the differences.

Methods: 50 users of combined oral contraceptive pill and 50 healthy controls were subjected to spirometric assessment in different phases of menstrual cycle. Student t test (2-tailed) was used to find the significance difference of PFT parameters between healthy controls and oral contraceptive user groups. Analysis of variance was used to find the significance of PFT for different phases of menstrual cycle in both the cases and controls.

Results: PEF was increased throughout the menstrual cycle in combined oral contraceptive pill users suggestive of bronchodilatory effect of progesterone. Within the group, both cases and controls showed increased FVC and FEVI suggestive of stimulation of ventilation by progesterone.

Conclusion: Subjects using combined oral contraceptive pills had improved lung function than controls when evaluated with computerized spirometer.

Key Words: $F E V_{1}$ as percentage of FVC $\left(F E V_{l} / F V C \%\right)$; Forced expiratory volume in 1 second $\left(F E V_{l}\right)$; Forced vital capacity (FVC); Peak expiratory flow (PEF), Premenstrual asthma (PMA)
\end{abstract}

\section{Introduction}

Menstrual cycle is an integral part of female reproductive phase. A normal menstrual cycle is dependent on the fluctuation in the ovarian hormones (estrogen and progesterone) during different phases of menstrual cycle - menstrual, follicular and luteal phases. The characteristic rhythmic changes in the rate of secretion of ovarian hormones produce corresponding changes not only in the reproductive system but in other organ systems as well. It is suggested that the lung function exhibit not only diurnal variations but also show changes dependent on different phases of menstrual cycle. ${ }^{1}$ Progesterone increases the ventilatory response of respiratory centre to $\mathrm{CO}_{2}$ and leads to reduced arterial and alveolar $\mathrm{PCO}_{2}$ in luteal phase. It is also a smooth muscle relaxant and a drop in plasma levels of progesterone in premenstrual days may result in bronchoconstriction due to the withdrawal of this relaxant effect on bronchial smooth muscle. This mechanism is proposed as a cause of premenstrual asthma (PMA) or menstrual-linked asthma. A subset of women with asthma reported worsening of their asthma symptoms few days before their menses and improvement of these changes with the abatement of their menses each month. ${ }^{2}$ Thus the diagnosis of premenstrual asthma is made upon the demonstration of "significant variation in airway function during the period just prior to the onset of menses". ${ }^{3}$ Up to $40 \%$ of female asthmatic subjects report a premenstrual deterioration in their condition which has been correlated with the estrogen and progesterone levels. ${ }^{4}$ Moreover, mortality due to asthma has increased to $59 \%$ among women versus $34 \%$ increase among men. ${ }^{5}$

The effect of corticosteroid hormones in asthma is well established but the effects of other steroid hormones have not been much investigated, despite evidences that they might be beneficial. In addition to being the most effective method of contraception, oral contraceptives have additional metabolic effects and provide many health benefits. This is because the combination oral contraceptives contain a potent, orally active progestin as well as an orally active estrogen and there is no time when the estrogenic target tissues are stimulated by estrogens without a progestin (unopposed estrogen). Oral contraceptives smoothen the hormonal fluctuations and improve pulmonary function thereby diminishing the airway hyper responsiveness. Hence, oral contraceptives are being investigated worldwide as a treatment for premenstrual asthma.

The dilemma in using oral contraceptives as an effective treatment for menstrual asthma arises from the conflicting reports which suggest that a continued rise in estradiol level post ovulation is the cause for improved ventilation and that exogenous administration of estrogen may improve peak expiratory flow rate and attenuate 
symptoms. It has also been proposed that oral contraceptive users have significantly higher total lung capacities when compared with non users during the follicular phase of menstrual cycle. ${ }^{6}$

Paradoxically, there are also scattered reports of premenstrual asthma patients in whom asthma becomes difficult to control during use of exogenous hormones. Discontinuation of hormone therapy in these patients has resulted in a return to baseline asthma control. ${ }^{7}$

All these emphasize the need for the clinician to be aware of the possible interactions between oral contraceptive pills and lung mechanics. More than 65 million in the world are estimated to be taking the "pill" of which about 9.52 million are estimated to be in India. Hence an attempt is made to study the effect of oral contraceptives on lung functions. In this study, it is intended to analyze the effects of oral contraceptives on respiratory function and compare the results with that of non contraceptive users.

\section{Material And Methods}

The study was conducted in Family Planning Clinics of Vani Vilas Hospital, Bangalore. A total of 100 women of age group $18-35$ years were selected for the present study. Of these, 50 women were taken as control and the other 50 women were in the study group. Inclusion criteria for the study group were women of age group 18 - 35 years taking an OCP for at least six months before the study, having regular menstruation, non-smoker and not taking any other medications. Subjects were excluded if they had any history of smoking, asthma, diabetes, hypertension, epilepsy, breast, ovary, or cervical cancer, or any other endocrine, metabolic or cardiovascular abnormalities. They were also excluded if they had been treated in the prior 4 weeks with oral steroids for any reason. 50 healthy volunteers of age group $18-35$ years who were not using oral contraceptive pills, with no history of asthma, dysmenorrhoea, dysfunctional uterine bleeding, menorrhagia, metrorrhagia, anaemia, cardiac disorders, tuberculosis, diabetes, smoking, obesity or psychiatric illness and have normal menstrual cycle of 28 - 30 days were taken and matched for age, sex, height, weight, BSA and considered as controls. Controls were excluded if they became pregnant. Thorough history was taken, including menstrual history and complete clinical examination was done before accepting them as subjects for the study. The subjects were selected after obtaining written, informed consent. Women with hormonal intra uterine device were not considered under exclusion criteria because of extensive studies showing that these devices have only local effects and no systemic effects. Subjects of study group were provided with a one-month supply of OCPs (MALA-N). The constituents per tablet of MALA-N used were levonorgestrel I.P. $0.15 \mathrm{mg}$ and ethinylestradiol I.P. $0.03 \mathrm{mg}$. Additionally, the brown tablets contained ferrous fumarate I.P. $60 \mathrm{mg}$ equivalent to ferrous iron $19.5 \mathrm{mg}$. An approval and clearance from the Institutional Ethics Committee was taken and the subjects were explained the design and purpose of the study. The subjects were rested thoroughly; thereafter, their height, weight and other personal details were recorded. Different subjects started their pulmonary function tests in different phases of menstrual cycle to rule out the training effect, if any.

Subjects were instructed to visit the laboratory for pulmonary function testing during each phase of menstrual cycle: $1-5^{\text {th }}$ day during menstrual phase, $9-12^{\text {th }}$ day during follicular phase and $19-22^{\text {nd }}$ day during luteal phase as estimated from their menstrual histories for the preceding 3 months. Phases were corroborated with the period of ovulation, as judged from daily basal oral temperature of the subject throughout the cycle.

Serum levels of estrogen and progesterone could not be estimated in the subjects due to financial constraints. The performance of the pulmonary function test was demonstrated after giving detailed instructions.

The subjects were made to undergo pulmonary function tests using the KIT MICRO RS 232C Computerized Spirometer for three times at every 15 minutes interval. The FVC, FEV 1 , PEF, and FEV $/ \mathrm{FVC}^{2}$ were recorded. The best of the three recordings was taken into account. All the tests were done at the same time of the day to avoid possible diurnal variations.

Statistical methods: Student $t$ test (2-tailed) was used to find the significance difference of PFT parameters between healthy controls and oral contraceptive user groups. Analysis of variance was used to find the significance of PFT for different phases of menstrual cycle in both the cases and controls.

Significance level interpretation (p: probability)

$\mathrm{p}<0.01 \quad$ highly significant (probability is $<1 \%$ )

$0.01<\mathrm{p}<0.05 \quad$ significant (probability $<5 \%$ )

$0.05<\mathrm{p}<0.10 \quad$ near significant

$0.10<\mathrm{p}<0.50 \quad$ possible trend but without significance

$\mathrm{p}>0.50 \quad$ no trend at all

\section{Result}

For ease of discussion, the menstrual phase was taken as days 1-5, follicular phase as days 9-12 and luteal phase as days 19-22 in the case group and the effect of oral contraceptives on different parameters of pulmonary function was compared with that of controls. Though FVC showed an increase in OCP users in the first 2 phases it was not statistically significant as compared to controls. $\mathrm{FEV}_{1}$ was increased in cases in the 
follicular phase as compared to controls but was not statistically significant. There was a highly significant difference in PEF in OCP users in comparison with the controls in all the 3 phases. (Table 1, 2, 3)

When the effect of oral contraceptives on PFT was studied overall in the different phases in the case group, FVC showed an increase in the luteal phase when compared to the menstrual and follicular phase (Table $4)$. The $p$ value at $<0.00$ indicates that this effect was highly significant. $\mathrm{FEV}_{1}$ was increased in the luteal phase as compared to the menstrual and luteal phase which was highly significant, $p$ value being $<0.00$. Though PEF was increased in luteal phase when compared to menstrual and follicular phase, this effect was not statistically significant, $\mathrm{p}$ value was $>0.40$. $\mathrm{FEV}_{1} / \mathrm{FVC} \%$ was not affected by any phase and was not statistically significant, $\mathrm{p}$ value was $>0.34$.

When the pulmonary function tests in different phases among the control group were studied as shown in table 5, FVC showed an increase in the luteal phase as compared to the menstrual and follicular phases which was statistically highly significant with $\mathrm{p}$ value $<0.00$. $\mathrm{FEV}_{1}$ was increased in the luteal phase as compared to the menstrual and follicular phase which was statistically highly significant with $p$ value $<0.01$. PEF was increased in the luteal phase as compared to the menstrual and follicular phase but was not statistically significant with $\mathrm{p}$ value $>0.40$. $\mathrm{FEV}_{1} / \mathrm{FVC} \%$ was unaffected by all the three phases and was not statistically significant with $\mathrm{p}$ value $>0.52$.

Table 1 Effect of OCPs on pulmonary function tests in menstrual phase

\begin{tabular}{|c|c|c|c|c|c|c|}
\hline \multirow{2}{*}{ Study variables } & \multicolumn{2}{|c|}{ Cases $(\mathrm{n}=50)$} & \multicolumn{2}{c|}{ Controls $(\mathrm{n}=50)$} & \multirow{2}{*}{$\begin{array}{c}\text { p-value } \\
\text { (2-tailed) }\end{array}$} \\
\cline { 2 - 5 } & Mean & SD & Mean & SD & & \\
\hline \multicolumn{7}{|c|}{ Recorded values } \\
\hline FVC & 2.53 & 0.20 & 2.47 & 0.33 & 1.04 & $>0.30$ \\
\hline FEV1 & 2.16 & 0.24 & 2.17 & 0.35 & 0.26 & $>0.80$ \\
\hline PEF & 6.24 & 1.27 & 4.79 & 1.82 & 4.62 & $<0.001$ \\
\hline FEV1/FVC\% & 0.85 & 0.07 & 0.88 & 0.08 & 1.91 & $>0.06$ \\
\hline
\end{tabular}

Table 2 Effect of OCPs on pulmonary function tests in Follicular phase

\begin{tabular}{|c|c|c|c|c|c|c|}
\hline \multirow{2}{*}{ Study variables } & \multicolumn{2}{|c|}{ Cases $(\mathrm{n}=50)$} & \multicolumn{2}{c|}{ Controls $(\mathrm{n}=50)$} & \multirow{2}{*}{$\begin{array}{c}\mathrm{p} \text {-value } \\
\text { (2-tailed) }\end{array}$} \\
\cline { 2 - 5 } & Mean & $\mathrm{SD}$ & Mean & $\mathrm{SD}$ & & \\
\hline \multicolumn{7}{|c|}{ Recorded values } \\
\hline FVC & 2.47 & 0.13 & 2.38 & 0.30 & 1.93 & $<0.06$ \\
\hline FEV1 & 2.13 & 0.12 & 2.08 & 0.34 & 1.01 & $>0.31$ \\
\hline PEF & 6.27 & 0.91 & 4.50 & 1.76 & 6.33 & $<0.001$ \\
\hline FEV1/FVC\% & 0.86 & 0.02 & 0.87 & 0.09 & 0.91 & $>0.36$ \\
\hline
\end{tabular}

Table 3 Effect of OCPs on pulmonary function tests in luteal phase

\begin{tabular}{|c|c|c|c|c|c|c|}
\hline \multirow{2}{*}{ Study variables } & \multicolumn{2}{|c|}{ Cases $(\mathrm{n}=50)$} & \multicolumn{2}{c|}{ Controls $(\mathrm{n}=50)$} & \multirow{2}{*}{$\begin{array}{c}\mathrm{p} \text {-value } \\
(2 \text {-tailed })\end{array}$} \\
\cline { 2 - 5 } & Mean & SD & Mean & SD & & $>0.97$ \\
\hline \multicolumn{7}{|c|}{ Recorded values } \\
\hline FVC & 2.67 & 0.14 & 2.67 & 0.37 & 0.04 & $>0.68$ \\
\hline FEV1 & 2.27 & 0.16 & 2.29 & 0.32 & 0.42 & $<0.001$ \\
\hline PEF & 6.51 & 1.02 & 4.98 & 1.79 & 5.25 & $>0.37$ \\
\hline FEV1/FVC\% & 0.85 & 0.05 & 0.86 & 0.09 & 0.91 & \\
\hline
\end{tabular}

Table 4 Comparison between different phases among cases

\begin{tabular}{|c|c|c|c|c|c|c|c|c|}
\hline \multirow{2}{*}{ Cases } & \multicolumn{2}{|c|}{ Menstrual phase } & \multicolumn{2}{|c|}{ Follicular phase } & \multicolumn{2}{|c|}{ Luteal phase } & \multirow{2}{*}{ F-value* } & \multirow{2}{*}{$\mathrm{p}$-value } \\
\cline { 2 - 11 } & Mean & SD & Mean & SD & Mean & SD & \\
\hline FVC & 2.53 & 0.20 & 2.47 & 0.13 & 2.67 & 0.14 & 22.31 & $<0.00$ \\
\hline FEV1 & 2.16 & 0.24 & 2.13 & 0.12 & 2.27 & 0.16 & 7.90 & $<0.00$ \\
\hline PEF & 6.24 & 1.27 & 6.27 & 0.91 & 6.51 & 1.02 & 0.93 & $>0.40$ \\
\hline FEV1/FVC\% & 0.85 & 0.07 & 0.86 & 0.02 & 0.85 & 0.05 & 1.09 & $>0.34$ \\
\hline
\end{tabular}

* F-value is obtained from ANOVA 
Table 5 Comparison between different phases among controls

\begin{tabular}{|c|c|c|c|c|c|c|c|c|}
\hline \multirow{2}{*}{ Control } & \multicolumn{2}{|c|}{ Menstrual phase } & \multicolumn{2}{|c|}{ Follicular phase } & \multicolumn{2}{|c|}{ Luteal phase } & \multirow{2}{*}{ F-value* } & \multirow{2}{*}{$\mathrm{p}$-value } \\
\hline & Mean & $\mathrm{SD}$ & Mean & SD & Mean & SD & & \\
\hline FVC & 2.47 & 0.33 & 2.38 & 0.30 & 2.67 & 0.37 & 9.90 & $<0.00$ \\
\hline FEV1 & 2.17 & 0.35 & 2.08 & 0.34 & 2.29 & 0.32 & 4.87 & $<0.01$ \\
\hline PEF & 4.79 & 1.82 & 4.50 & 1.76 & 4.98 & 1.79 & 0.93 & $>0.40$ \\
\hline FEV1/FVC\% & 0.88 & 0.08 & 0.87 & 0.09 & 0.86 & 0.09 & 0.66 & $>0.52$ \\
\hline
\end{tabular}

* F-value is obtained from ANOVA

\section{Discussion}

The pathogenesis of premenstrual asthma is not fully understood and a rapid fall in progesterone level during the late luteal phase causing bronchoconstriction has been proposed as the possible mechanism. Matsuo et al. had concluded that maintenance of stable levels of sex hormones and the disappearance of cyclic changes in their levels by administration of oral contraceptives help in the improvement of condition. ${ }^{8}$ Dimple Arora et al. observed that mean values of all lung functions i.e., $\mathrm{FEV}_{1}, \mathrm{PEFR}, \mathrm{FEF}_{25-75 \%}$ were significantly higher in secretory phase followed by follicular phase and lowest in menstrual phase in asthmatic women and concluded that the most possible cause for this increase is the hyperventilation associated with increased levels of progesterone secretion in this phase. ${ }^{9}$ In the present study there was a significant increase in PEFR in OCP users when compared to the controls. When the effect of oral contraceptives on pulmonary function tests was studied overall in the different periods of menstrual cycle in the case group, FVC and $\mathrm{FEV}_{1}$ showed an increase in the luteal phase which is highly significant as compared to menstrual and follicular phase but the same effect was also seen in the controls. Peak expiratory flow reflects mainly the calibre of the bronchi. Progesterone reduces constriction of the airways and relaxes the bronchial smooth muscle in the airways by activating $\beta_{2}$ adrenergic receptors. By maintaining progesterone levels throughout the menstrual cycle and increasing PEFR, OCPs may prevent the exacerbation of asthma. The unchanged FVC, FEV 1 during each phase reflected the unchanged mechanical properties of lungs during menstrual cycle.

The present study was in agreement with Resmi S.S et al. who studied the effects of OCPs on respiratory function and showed an increase in PEFR. ${ }^{10}$ However, they observed a significant decrease in $\mathrm{FEV}_{1} / \mathrm{FVC} \%$ among oral contraceptive users as compared to controls which was not found in this present study.

The present study is partly in agreement with Pradeep Kumar et al. who studied the effects of OCPs on airway resistance in OCP users and non users and showed a significant increase in $\mathrm{FEF}_{25-75 \%}$ and PEFR in OCP users. ${ }^{11}$ They concluded that hormonal pills decrease the resistance offered by small bronchioles.

Orally administered synthetic progesterone in doses found in current OCPs is a moderate stimulator of ventilation in normal healthy women. Since steroids easily cross the blood-brain barrier, they act on central receptors. A proposed mechanism for the action of synthetic progestins is that their administration may play a role in the central amplification of neural input from peripheral chemo receptors which in turn has an important influence on cholinergic outflow of airways which may cause reflex increase in ventilation. The contraceptive pills contain synthetic form of progesterone which are highly reactive than natural progesterone.

The study also agrees partly with Mamoona Shafiq et al. who observed a significant increase in FVC and PEFR after use of OCPs for 6 months. ${ }^{12}$ However, they concluded that the observed improvement in lung mechanics was due to estrogen and not due to progesterone and that estrogen could be intensifying the effect of progesterone in humans.

Montes. A et al and Das A.K et al did not find appreciable changes in the functions in OCP users in their studies. ${ }^{13,14}$ The present study contradicts these works.

\section{Conclusion}

OCPs maintain a constant endocrine profile over the entire menstrual cycle and continued progesterone support probably has a role in preventing premenstrual drop of lung volumes and capacities. Physicians treating women with premenstrual asthma need to be aware of the potential benefits of oral contraceptive pills in treating the same.

\section{Limitations and issues for future study:}

The present study was done on healthy cases using oral contraceptive pills. A well designed prospective study where the women diagnosed with premenstrual asthma themselves act as controls and their follow-up after giving oral contraceptive pills with measurement of pulmonary function parameters longitudinally over the course of several menstrual cycles could determine the consistency of the relationship. 


\section{References}

[1] C. S. Rajesh, Gupta P, Vaney N. Status of pulmonary function tests in adolescent females of Delhi. Indian J Physiol Pharmacol 2000; 44 (4): 442-448

[2] Skobeloff E.M, Spivey W.H, Silverman R et al. The effect of the menstrual cycle on asthma presentations in the emergency department. Arch Intern; 156:1837-40.

[3] Agrawal A.K, Shah A. Menstrual-linked asthma. J Asthma 1997; 34: 539-45.

[4] Rumball J. Understanding the relationship between asthma and the menstrual cycle. The Western Journal of Graduate Research $2001 ; 10(1) 65-68$.

[5] de Marco R, Locatelli F, Sunyer J, Burney P. Differences in incidence of reported asthma related to age in men and women. A retrospective analysis of the data of the European Respiratory Health Survey. Am J Respir Crit Care Med 2000; 162: 68-74.

[6] Caracta C. F. Gender differences in pulmonary disease. The Mount Sinai Journal of Medicine 2003; 70(4): $215-17$.

[7] Derimanov S.G, Oppenheimer J. Exacerbation of premenstrual asthma caused by an oral contraceptive. Ann Allergy Asthma Immunol 1998; 81: 243-46.

[8] Matsuo N, Shimoda T, Matsuse et al. A case of menstruation-associated asthma. Chest 1999; 116: 252-53.

[9] Arora D, Kaur P, Arora M, Gupta U. Influence of menstrual cycle on lung functions in asthmatic females. Indian Journal of Fundamental and Applied Life Sciences ISSN: 2231-6345 2011 vol. 1(4): 62-7.

[10] Resmi S.S., Samuel E, C. Kesavachandran et al. Effect of oral contraceptives on respiratory function. Indian J Physiol Pharma col 2002; 46 (3): 361-66.

[11] Kumar P, Singh S, Singh U, Verma P. Oral contraceptive pills decrease pulmonary airway resistance in healthy north Indian women. Indian J Med Sci 2011; 65: 64-8.

[12] Shafiq M, Sheikh S A, Hassan S H. Combined oral contraceptive pills improve lung function variables. Pak J Physiol 2012; 8 (1).

[13] Montes A, Lally D, Hale R.W. The effects of oral contraceptives on respiration. Fertil Steril 1983; 39 (4): $515-19$.

[14] Das A.K, Majumdar, Singh A.M. A study of extragenital effects of estrogen and progesterone. Indian J Physiol Pharmacol 2002; 46 (2): $175-85$ 